\title{
Novel Dual Band-Reject Microwave Filter Using $C$-Shaped Defected Ground Structures
}

\author{
Mostafa Attaran Kakhki and Mohammad H. Neshati
}

\begin{abstract}
In this paper, a new compact dual band-reject $C$-shaped defected ground structure (DGS) for microstrip line is introduced. Using two units of the same shaped DGS with different dimensions, a dual band-stop filter is designed and implemented. Results show that there are two parallel resonance frequencies at $2.468 \mathrm{GHz}$ and $6.629 \mathrm{GHz}$ with a very high $Q$-factor. The frequency characteristics of the proposed filter could be controlled by adjusting the DGS parameters such as width and length of both $C$-shaped patterns. Also, the distance between the two DGS structure could be adjusted for the required frequency characteristics. The proposed DGS provides $Q$-factor of more than $\mathbf{5 0}$ with attenuation of at least 20 $\mathrm{dB}$ at the resonant frequencies. The new introduced DGS filter is successfully designed and fabricated and the measured results are in a very good agreement with those obtained by numerical investigation.
\end{abstract}

Index Terms-Defected ground structure (DGS), dual bandreject filter, $Q$ factor.

\section{INTRODUCTION}

Defected Ground Structures (DGSs) was introduced by J. I. Park et al in 1999. These novel structures which found on Photonic Band-gaps (PBGs), could be applied in design of microwave planner circuits such as filters. The most important feature of DGS structures is compatible with monolithic Microwave Integrated Circuits (MICs) and also planner mirostrip antennas [1]-[3].

The grounding current distribution of a mirostrip circuit could significantly changed by a periodic or a non-periodic etching of a patterned ground structure leading to altering the transmission line parameters and so, controlling the frequency response of the circuits. Traditional PBG structures utilize periodical array of cells, while DGSs use only one defected pattern providing band-reject or band-pass characteristics. In turn, DGSs are compact, small in size and adapt to miniaturizing of planner microwave circuits. Moreover, DGS structures can be modeled by simple resonant circuits and so their parameter extraction is simple, offering advantageous in the design of microwave and millimeter-wave circuits [4]-[11].

In this paper, a new dual $C$-shaped DGS filter is introduced. The proposed filter provides two parallel resonance frequencies at $2.4 \mathrm{GHz}$ and $6.7 \mathrm{GHz}$. At both frequencies the obtained band-stop bandwidth is low, leading to a measured $Q$-factor of 65 at first resonance and 61.3 at the second one.

Manuscript received October 14, 2012; revised November 25, 2012.

Mostafa Attaran Kakhki is with Electrical Department of Ferdowsi University of Mashhad, Mashhad, Iran (e-mail: m.attarankakhki@ieee.org).

\section{Duall Band-Reject Dgs Filter Structure}

The 3-D of the proposed is shown in Fig. 1. The DGS filter consists of two $C$-shaped patterned ground structure with different dimensions resonating at two distinct frequencies. $3-\mathrm{D}$ view of the proposed filter is shown in Fig 1-a. Also, the unit cell of the structure with defined cell dimensions is shown in Fig 1-b. All details of the cell sizes are summarized in Table 1. The line width is chosen for the microstrip line impedance of $50 \Omega$. RF-35 PC-board with a relative dielectric constant of 3.5 and thickness of 60 -mil, $1.524 \mathrm{~mm}$, is used as the substrate of the microstrip structure and PC-board size is $30 \times 40 \mathrm{~mm}^{2}$.

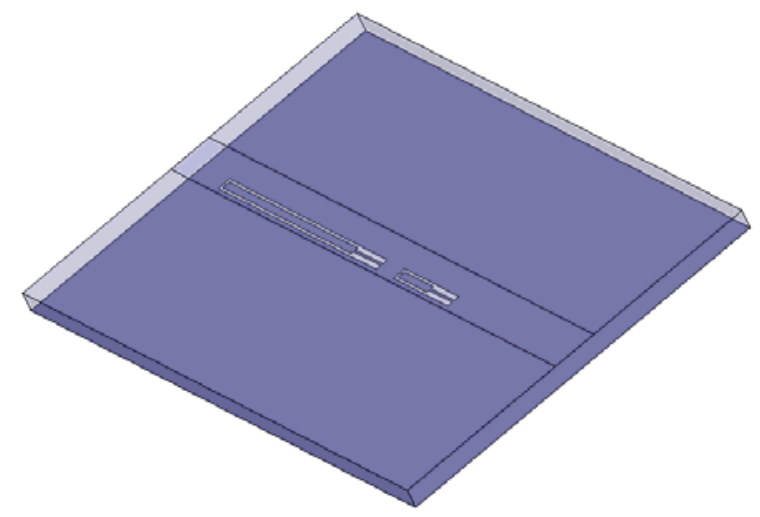

(a)

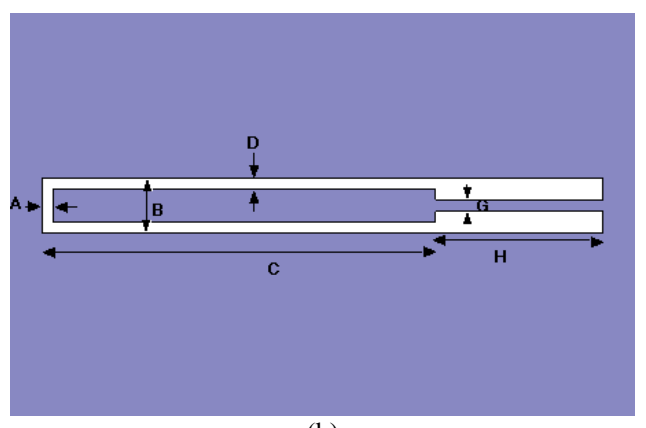

(b)

Fig. 1. Geometry of the proposed C-Shaped DGS filter:, a) 3-D view, b) unit cell.

\section{Simulation Results}

The proposed filter under investigation is numerically examined using HFSS. First, different cells are considered, their return loss and forward transmission are carried out. For the first cell, $S_{11}$ and $S_{12}$ are plotted in Fig. 2 versus frequency. It can be seen that resonance frequency is at $2.42 \mathrm{GHz}$ with rejection bandwidth of $20 \mathrm{MHz}$. The simulated $Q$-factor of this cell is 121 .

The second cell is going to have rejection characteristics at 5.7 GHz. The dimensions of the first cell are changed and 
optimized having an acceptable high $Q$-factor at the second resonance frequency. The results are summarized in Table 1. Fig. 3 shows $S_{11}$ and $S_{12}$ versus frequency. It can be seen that band-rejection property of $31 \mathrm{~dB}$ at $5.69 \mathrm{GHz}$ is provided by $3-\mathrm{dB}$ bandwidth of $36 \mathrm{MHz}$ and $Q$ - factor at this resonance is 158.

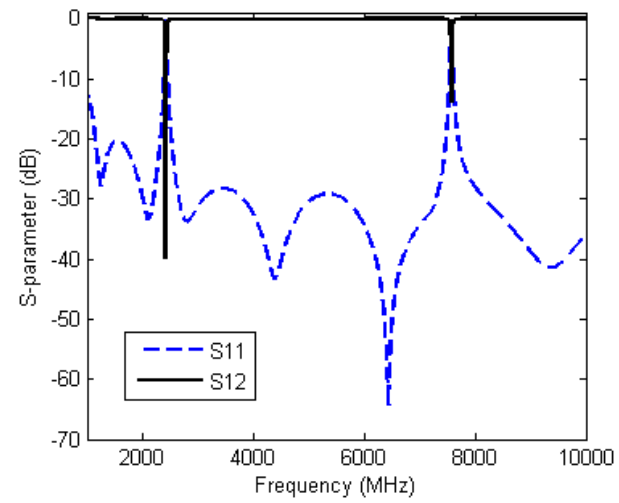

(a)

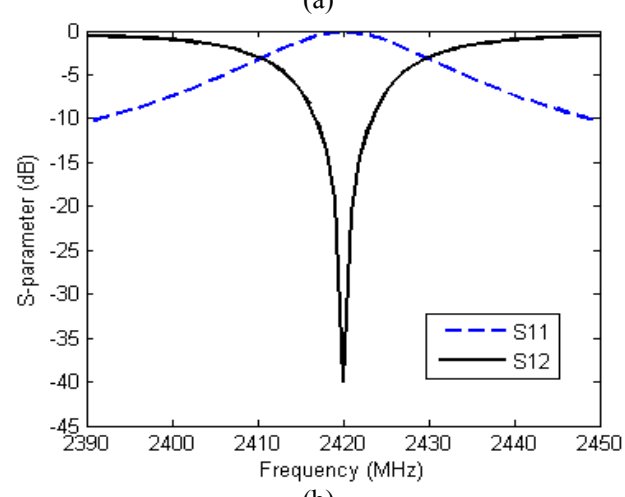

(b)

Fig. 2. S-parameters of first $C$-shaped DGS cell 1: a) form $1 \mathrm{GHz}$ to $10 \mathrm{GHz}$, b) around resonance frequency.

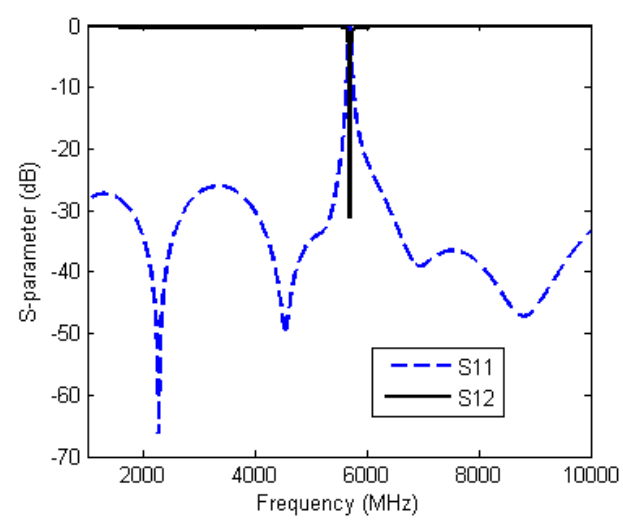

(a)

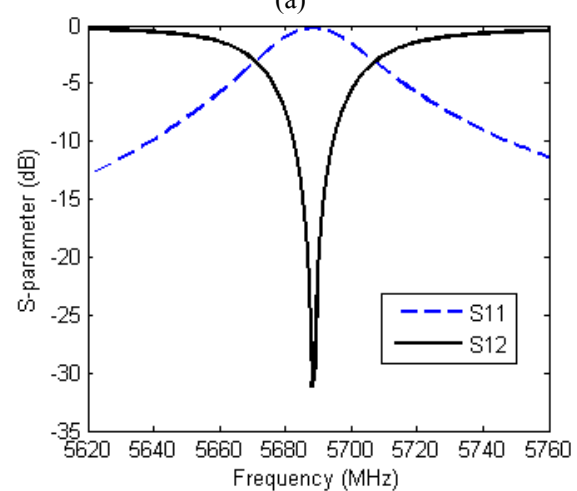

(b)

Fig. 3. S-parameters of first $C$-shaped DGS cell 2: a) form $2 \mathrm{GHz}$ to $10 \mathrm{GHz}$, b) around resonance frequency.
TABLE I: CELL DimENSIONS OF THE DUAL C-ShAPED DGS FILTER

\begin{tabular}{|c|c|c|}
\multicolumn{3}{c}{ (ALL DIMENSION IN MM) } \\
\hline & cell 1 $(2.4 \mathrm{GHz})$ & cell $2(5.7 \mathrm{GHz})$ \\
\hline$A$ & 0.2 & 0.2 \\
\hline$B$ & 1 & 1 \\
\hline$C$ & 14.2 & 3.4 \\
\hline$D$ & 0.2 & 0.2 \\
\hline$G$ & 0.2 & 0.2 \\
\hline$H$ & 3 & 2.5 \\
\hline
\end{tabular}

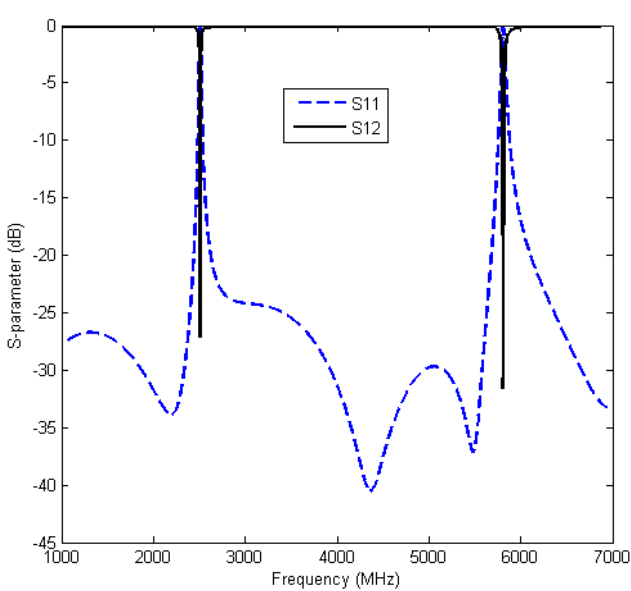

(a)

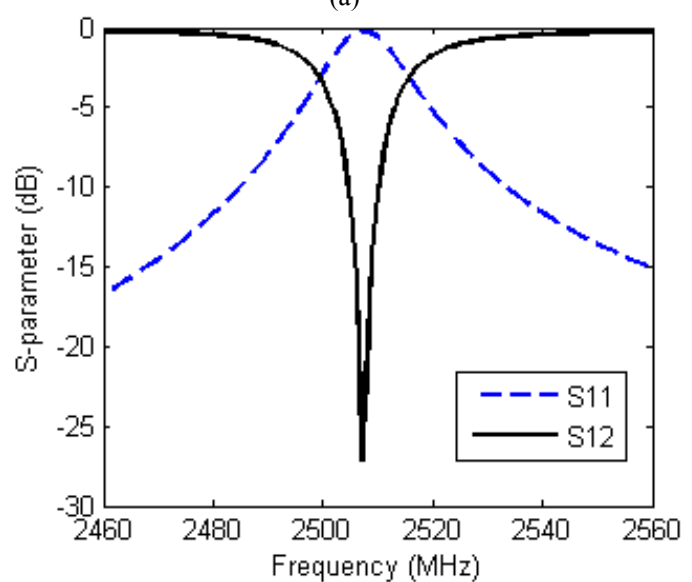

(b)

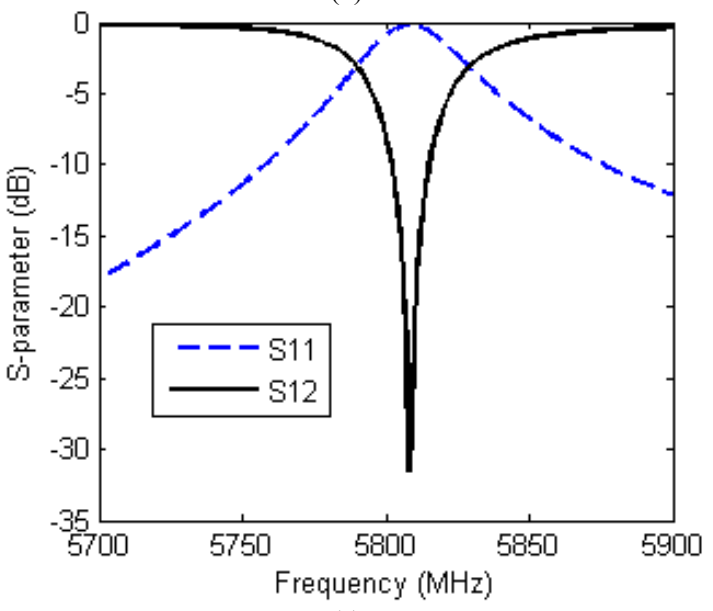

(c)

Fig. 4. S-parameters of dual band-reject $C$-shaped DGS: a) form $1 \mathrm{GHz}$ to $7 \mathrm{GHz}, \mathrm{b}$ ) around the first resonance, $\mathrm{c}$ ) around the second resonance.

\section{DuAl BAND REJECT DGS FILTER DESIGN}

To obtain a dual band-reject filter, two $C$-shaped DGSs designed in previous section are placed in cascade as shown in Fig. 1-a. The rejection characteristics of the dual-band 
filter are not only depending on the dimensions of the each cell, but also the distance between the two cells affects the frequency response of the filter.

First of all, the distance between the two cells was set to $\delta=1.9 \mathrm{~mm}$ and filter was examined by HFSS. Fig. 4 -a shows the $S$-parameters of the DGS structure. The detailed frequency response around the two resonances is plotted in Fig. 4-b and Fig. 4-c. It can be seen that the two resonance frequencies are at $2.507 \mathrm{GHz}$ and $5.808 \mathrm{GHz}$. Cascading the cells causes that resonance frequency of individual cells changes from $2.42 \mathrm{GHz}$ and $5.69 \mathrm{GHz}$ to the new resonance frequencies of $2.507 \mathrm{GHz}$ and $5.808 \mathrm{GHz}$ respectively. As the frequency shift is not too much, it can be concluded that resonance frequencies can be controlled independently by adjusting the size of each DGS cell.

Moreover, a parametric study was carried out and $S$-parameter of the dual cell $C$-shaped DGS filter was numerically investigated versus $\delta$, the distance between the two cells. The simulation results of $S$-parameters for various $\delta$ from $1.4 \mathrm{~mm}$ to $2.9 \mathrm{~mm}$ with $0.5 \mathrm{~mm}$ steps are shown in Fig. 5 . It can be concluded that increasing the distance between cells lead to lowering resonance frequency and rejection bandwidth of the first resonant frequency. For the second resonant frequency, increasing $\delta$ foe mentioned range of variation; resonance frequency is decreased, while no significant changes can be seen in rejection bandwidth.

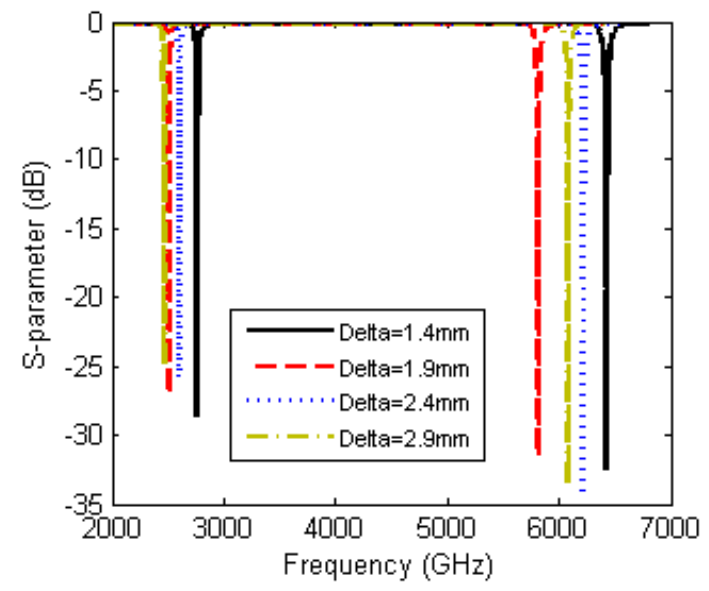

Fig. 5. S-parameters of dual band-reject $C$-shaped DGS: a) form $1 \mathrm{GHz}$ to $7 \mathrm{GHz}, \mathrm{b}$ ) around the first resonance, c) around the second resonance.

\section{EXPERIMENTAL RESUlts OF DUAl BANDGAP FILTER}

To verify the simulation results, the proposed filter was implemented and $S$-parameters have been measure using HP8510 network analyzer. The dimensions of the cells are shown in Table 1 and the distance between cells is set to 1.9 mm. Fig. 6 shows bottom view of the fabricated filter.

In Fig. 7-a. measured $S_{11}$ and $S_{12}$ are plotted versus frequency. It can be seen that the filter not only exhibits a slow wave characteristic with low insertion loss, but also provides a sharp frequency response at stop-band. Return loss is less than $-17 \mathrm{~dB}$ over the flat pass-band.

Two resonance frequencies are at $2.42 \mathrm{GHz}$ and $6.65 \mathrm{GHz}$. At the first resonant, rejection bandwidth is $38 \mathrm{MHz}$ with a $Q$-factor of 65 nearly half of the simulation results. The rejection bandwidth is $108 \mathrm{MHz}$ with a $Q$-factor of 61.3 for the second resonant.

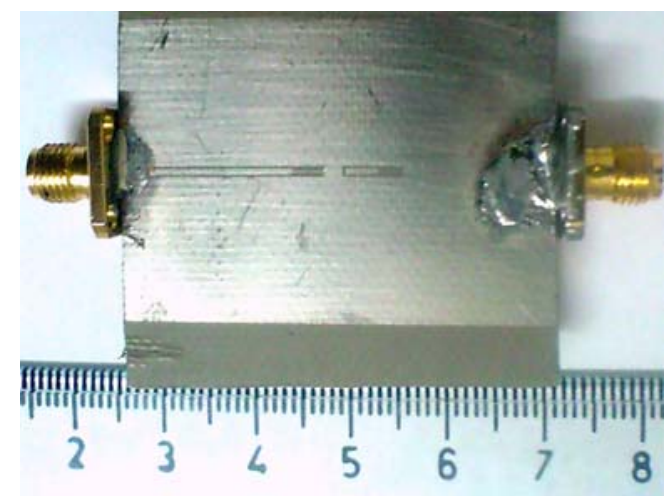

Fig. 6. Bottom view of the fabricated filter.

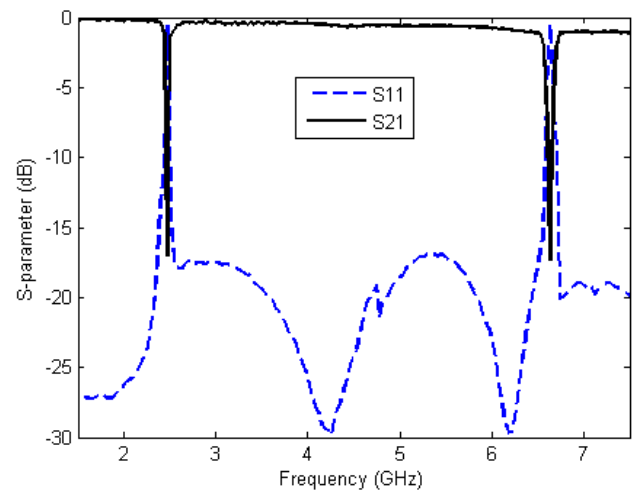

(a)

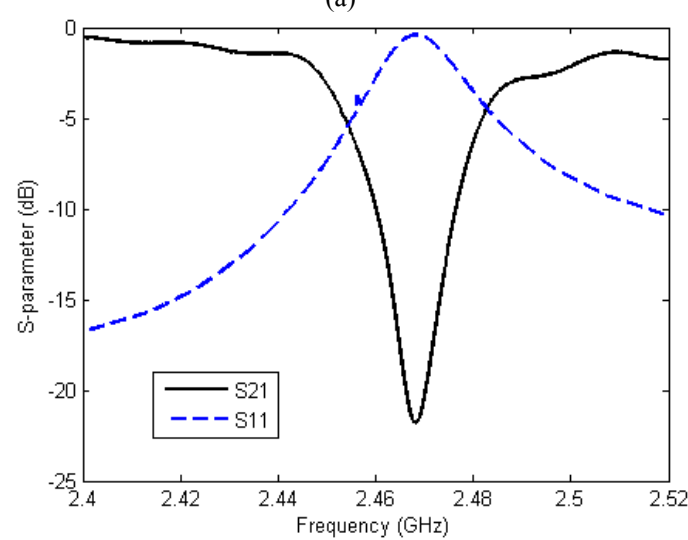

(b)

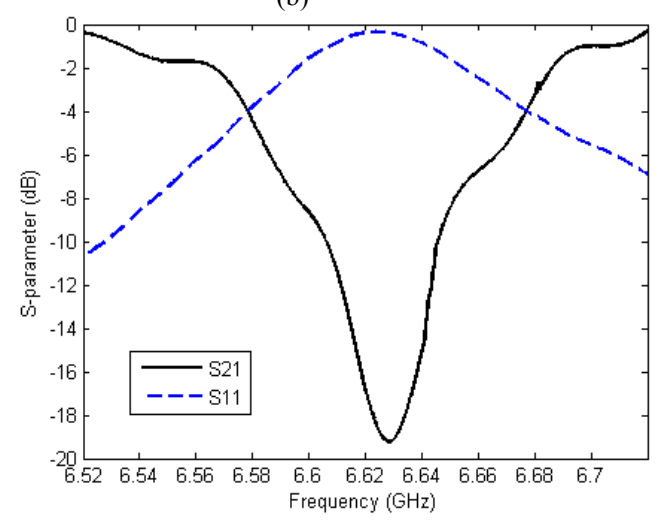

(c)

Fig. 7. Measured S-parameters of the fabricated filter: a) form $1.5 \mathrm{GHz}$ to $7.5 \mathrm{GHz}, \mathrm{b})$ around the first resonance, $\mathrm{c}$ ) around the second resonance.

Both rejection bandwidths have very sharp cutoff frequency response. The suppression for the first resonance frequency is approximately $22 \mathrm{~dB}$, while for the second resonant frequency; the suppression is around $19 \mathrm{~dB}$. Apart 
from an error for the second resonance frequency, other measured are agree well with simulation results. It is said this is mainly due to $t$, the finite size of ground, tolerances of cells dimensions and also one cell is fabricated near the measured port.

\section{CONCLUSION}

In this paper, a novel miniature dual $C$-shaped DGSs with improved $Q$-factor have been introduced. The proposed filter is numerically investigated by HFSS. By adjusting the distance between the cells, two measured resonance frequency at $2.42 \mathrm{GHz}$ and $6.65 \mathrm{GHz}$ is obtained. The first resonance exhibits a $Q$-factor of 65 while the second resonance $Q$-factor is 63.1 . The attenuation of the two resonance frequencies are $-22 \mathrm{~dB}$ and $-20 \mathrm{~dB}$ respectively. Apart from an error in measuring the second resonance frequency, other measured parameters agree well with those obtained by simulation. Providing an acceptable attenuation around the resonance frequencies, the proposed dual band-reject filter has small size and narrow band width and also has multi-band rejection property. This kind of filter should be used in the design of microwave circuits, antenna arrays, where suppression of multiple frequency bands and miniaturization of the circuits are needed.

\section{ACKNOWLEDGMENT}

The authors would like to appreciate Iran Telecommunication Research Center (ITRC) for their financial support.

\section{REFERENCES}

[1] J. S. Park and M. S. Jung, "A novel defected ground structure for an active device mounting an application to a microwave oscillator," IEEE Microw. Wireless Compon. Lett., vol. 14, no. 5, pp. 198-200, May 2004.

[2] D. J. Woo and T. K. Lee, "Suppression of harmonics in Wilkinson power divider by using asymmetric spiral DGS," in Proc. of 34 th Eur. Microwave Conf. Dig., vol. 1, Oct. 2004, pp. 467-470.

[3] C. S. Kim, J. S. Park, D. Ahn, and J. B. Lim, "A novel 1-D periodic defected ground structure for planar circuits," IEEE Microw. Guided Wave Lett., vol. 10, no. 4, pp. 131-133, Apr. 2000.

[4] D. J. Woo, T. K. Lee, J. W. Lee, C. S. Pyo, and W. K. Choi, "Novel U-slot and V-slot DGSs for bandstop filter with improved Q factor," IEEE Trans. Microw. Theory Tech., vol. 54, no. 6, pp. 2840-2847, Jun. 2006.
[5] J. S. Park, J. S. Yun, and D. Ahn, "A design of the novel coupled-line bandpass filter using defected ground structure with wide stopband performance," IEEE Trans. Microw. Theory Tech., vol. 50, no. 9, pp. 2037-2043, Sep. 2002.

[6] J. S. Lim, Y. C. Jeong, D. Ahn, and S. W. Nam, "A technique reducing the size of microwave amplifiers using spiral-shaped defected ground structure," J. Korea Electromagn. Eng, vol. 14, no. 9, pp. 904-911, Sep. 2003.

[7] D. Ahn, J. S. Park, C. S. Kim, J. N. Kim, Y. Qian, and T. Itoh, "A design of the low-pass filter using the novel microstrip defected ground structure," IEEE Trans.Microw. Theory Tech., vol. 49, no. 1, pp. 86-93, Jan. 2001.

[8] K. H. Yi and B. K. Kang, "Modified Wilkinson power divider for $n$th harmonic suppression," IEEE Microw. Wireless Compon. Lett., vol. 13, no. 5, pp. 178-180, May 2003.

[9] D. J. Woo and T. K. Lee, "Suppression of harmonics in Wilkinson power divider using dual-band rejection by asymmetric DGS," IEEE Trans. Microw. Theory Tech, vol. 53, no. 6, pp. 2139-2144, Jun. 2005.

[10] J. S. Lim, C. S. Kim, D. Ahn, and Y. C. Jeong, "Design of low-pass filter using defected ground structure," IEEE Trans. Microw. Theory Tech, vol. 53, no. 8, pp. 2539-2545, Aug. 2005.

[11] S. Y. Huang and Y. H. Lee, "A compact E-shaped patterned ground structure and its applications to tunable bandstop resonator," IEEE Transactions on Microwave Theory and Techniques, vol. 57, no. 3, pp. 657-666, Mar., 2009.

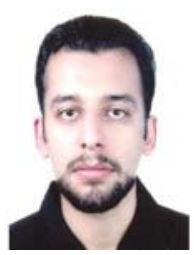

Mostafa Attaran Kakhki was born in Mashhad, Iran, in 1984. He received the B.Sc. degree in electrical engineering from Islamic Azad University of Central Tehran Branch (IAUCTB) and his M.Sc. degree of electrical engineering at Ferdowsi University of Mashhad, Mashhad, Iran. His research interests are microstripe antennas and filters and DGS structures

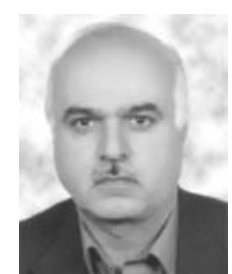

Mohammad H. Neshati was born in Yazd, Iran. He received the B.S. degree from Isfahan University of Technology, Isfahan, Iran, in 1984, the M.S. degree from Amir Kabir University of Technology, Tehran, Iran, in 1988, and the Ph.D. degree from the University of Manchester Institute of Science and Technology (UMIST), Manchester, U.K., in 2000, all in electrical engineering. In 1889, he joined the faculty of engineering at the University of Sistan and Baluchistan, Zahedan, Iran, where he taught electromagnetics, field theory, microwave circuits and conducted research in the development of microwave antennas especially on dielectric resonator antennas (DRAs). He recently joined the Electrical Engineering Department, Ferdowsi University of Mashhad, Mashhad, Iran, as an Assistant Professor here he conducts research in EM theory, antennas and microwave circuits. 